\title{
Complications of Transurethral Resection of the Prostate
}

\author{
Albert Pesha \\ Service of Urology, Regional Hospital Fier, Fier, Albania \\ Email address: \\ albertpesha@hotmail.com
}

\section{To cite this article:}

Albert Pesha. Complications of Transurethral Resection of the Prostate. Clinical Medicine Research. Vol. 5, No. 3, 2016 , pp. $24-27$. doi: $10.11648 /$ j.cmr.20160503.12

Received: March 24, 2016; Accepted: April 18, 2016; Published: April 28, 2016

\begin{abstract}
Benign prostatic hypertrophy can be treated by several therapeutic options including medical and surgical techniques. Among the surgical options, transurethral resection has a crucial role. Transurethral resection of the prostate (TURP) remains the standard surgical treatment for benign prostatic hypertrophy (BPH). This technique was the first minimally invasive procedure of the modern surgical practice remaining the most commonly procedure. During 2010-2015, 324 patients were enrolled in our study, recording all postoperative complications. Our paper emphasizes all the theoretical aspects of TURP's complications comparing to our data.
\end{abstract}

Keywords: Prostate Gland, Transurethral Resection, Bleeding, Hyponatremia

\section{Introduction}

Transurethral resection of the prostate (TURP) has a played a significant role in modern era of benign prostatic hypertrophy treatment. This minimally invasive procedure can reduce the complications comparing to open surgery, reduces hospital length and costs, and finally reducing complications can enhance the quality of life [1]. TURP is recently considered as a gold standard besides several other alternatives as YAG laser [2], ablation, and KTP-laser photoselective vaporization $[3,4]$. The indications of TURP include benign prostate hypertrophy, bladder calculi, bladder outlet obstruction (infection, hematuria, and renal failure due to obstruction). Transurethral resection of prostate remains the standard technique of surgical management of BHP, nevertheless of several surgical alternatives [5]. Perhaps there is no absolute contraindication, but a careful evaluation is advisable when high risk patient and coagulopathy are present. Our aim was to identify the complications after TURP in our series comparing with the other studies.

\section{Material and Methods}

This is a retrospective describing study performed close Service of Urology in Regional Hospital Fier in Albania. The study took place in the period 2010-2015. There were enrolled 324 patients, scheduled to undergo transurethral resection of prostate for prostate enlargement and medically resistant. There were excluded all the patients suffering from malignant form of prostate diseases. The patients were evaluated by a multidisciplinary team including nephrologists, anesthesiologist and the surgeon. The preoperative diagnostic tests were ultrasound examination, prostatic antigen measurement, transrectal ultrasound examination, cardio-respiratory screening, and biochemistry tests. The patients were admitted to the hospital the morning of surgery. An oral quinolon was used for prophylactic purposes. The problematic patients were admitted $24 \mathrm{~h}$ before surgery and endovenous antibiotic's route of administration is established as well.

We used Storz monopolar device, a resectoscope $26 \mathrm{Fr}$, and 30 degrees optic tool. The irrigation fluid was glucose $5 \%$. After the resectoscope was inserted, the resection begun from median lobe and then the lateral lobes were resected. We tried to expand the resection field up to vero-montanum in order to prevent the external sphincter injury, which can induce the postoperative total incontinence. Several times we applied a suprapubic catheter in order to shorten the surgery time and minimizing the possibility of TURP syndrome. After the prostatic gland removal was completed, a 20 or 22 Fr urinary catheter is inserted with $30 \mathrm{ml}$ balloon inflated. The urinary catheter was maintained in traction position for approximately $3 \mathrm{~h}$ after the surgery. Next 24 hours the urinary bladder was irrigated by normal saline fluid. Urinary 
catheter was removed approximately in the fourth postoperative day judging case by case.

There were recorded all the demographic parameters of the patients, the size of enlarged prostate, residual urine amount, resection time, and finally the post transurethral resection complications. Table 1 summarizes all the preoperative findings and demographic data.

Table 1. Preoperative findings and demographic data.

\begin{tabular}{ll}
\hline Parameters & Number \\
\hline Total number of patients $(\mathrm{n})$ & 324 \\
Average age (years old) & $70.8 \pm 5.6$ \\
Volume of prostate $\left(\mathrm{cm}^{3}\right)$ & $62.26 \pm 7.6$ \\
Residual urine amount $(\mathrm{ml})$ & $205 \pm 34.7$ \\
\hline
\end{tabular}

\section{Results}

As afore mentioned there were recorded resection time and posturethral resection complications. The resection time was evaluated $65 \pm 13.5$ minutes. The faced complications were bleeding, perforation of prostatic capsule, external urethral sphincter injury, urethral stricture, bladder neck stenosis, retrograde ejaculation, detrussor muscle injury, infection complications (sepsis, epididimitis), and finally the mortality rate. There were recorded no death or TURP syndrome in our serial.

Table 2. The complications of intra/postoperative period.

\begin{tabular}{ll}
\hline Complication & Percentuality \\
\hline Intraoperative bleeding & $4.62 \%$ \\
Postoperative bleeding & $4.9 \%$ \\
Blood transfusion rate & $3.55 \%$ \\
Prostate capsule perforation & $8.1 \%$ \\
Intraperitoneal irrigated fluid & $1.6 \%$ \\
Total urinary incontinence & $0.35 \%$ \\
Early urge incontinence & $40 \%$ \\
Retrograde ejaculation & $95 \%$ \\
Urinary tract infection & $39 \%$ \\
Epididymitis & $7.11 \%$ \\
Urinary retention & $4.98 \%$ \\
Re-TURP & $3.2 \%$ \\
Urethral stricture & $10.2 \%$ \\
Bladder neck stenosis & $0.7 \%$ \\
\hline
\end{tabular}

Bleeding is a major concern of this technique. We encountered bleeding as intraoperative $(4.62 \%)$ and postoperative bleeding $(4.9 \%)$. The blood transfusion rate was approximately $3.55 \%$. The prostate capsule perforation occurred in 23 patients (8.1\%). Intraperitoneal irrigation fluid had an incidence of $1.6 \%$. No TURP syndrome was verified as well. External sphincter injury can result in stress or total urinary incontinence, decreasing the quality of life. Total urinary incontinence was evidenced at only one patient, whereas early urge incontinence occurred up to $40 \%$ of the patients. Retrograde ejaculation occurred in $95 \%$. Urinary tract infection presented in 39\% whereas epididymitis was faced in $7.11 \%$. In the postoperative period, urinary retention had an incidence of $4.98 \%$. Re-TUR procedure was performed in $3.2 \%$ of the patients. Urethral stricture was encountered in $10.2 \%$, and bladder neck stenosis at 2 patients $(0.7 \%)$. Table 2 includes all the postoperative findings and complications.

\section{Discussion}

The transurethral resection of prostate remains the standard technique of surgical management of BHP, nevertheless of several surgical alternatives [5]. Indications include bladder obstruction, urinary retention, bladder calculi, and hematuria [6]. The complications are patients related and surgical related. The patient's related complications are cardiac arrhythmia, myocardial infarction, pulmonary embolism, preexisting respiratory diseases exacerbation, deep venous thrombosis, and death. The surgical related complication includes clot retention, bleeding, capsular perforation, urosepsis, incontinence, and urinary tract infections.

In our serial we memorized all the complications, in order to understand our performance and the discrepancies in comparison with other studies. Bleeding was our main concern because of being one of the main TURP complications. Bleeding can be due to venous and/or arterial origin. Venous bleeding is primarily caused by venous sinuses damage and capsular perforation. Arterial bleeding is infection or congestion related. Several authors reported that anti-androgenic therapy can be a suitable option for minimizing the inraoperative bleeding [7]. The bleeding origin can be determined by flow and optic direction relationship (arterial bleeding is directed to the optic), and the anatomical position (being close to the apex) [7]. Rarely the bleeding is due to fibrinolysis secondary to plasmin production. It is well known that prostatic tissue is rich of plasminogen activator and thromboplastine. The endoscopic coagulation and balloon compression are the main steps to minimize bleeding and must be done carefully in order to indentify the hidden sources. The reported incidence of bleeding following TURP is from $0.4 \%-22 \%$. Our bleeding incidence ranged $4.62 \%-4.9 \%$ with a transfusion rate of $3.55 \%$, which are comparable values with the other studies, despite our relatively little experience in TURP interventions. The arterial bleeding is dominated in operating room, whereas the venous bleeding is more difficult to be successfully treating and mainly in postoperative period. If bleeding becomes difficult to be controlled, the surgeon must terminate the procedure as soon as possible and a Foley urinary catheter must be inserted and maintained in traction. In our institution during bleeding episodes, we try to identify the origin and the position by reducing the irrigation flow. In our serial we faced 6 patients $(1.85 \%)$ whom undergone in reintervention within 2 days after the first surgery but no bleeding source was indentified. The later re-TURP was performed in $3.2 \%$ of all cases mainly due to patients related late complains.

TURP syndrome is one of the major complications due to extravasations, hyperglycemia, and surgical time. The pathphysiological mechanism includes hypervolemic hyponatremia, hyperglycemia due to irrigation fluid type, venous sinuses damage, and operating time. The main 
mechanism is of course hypervolemic hyponatremia which induces myocardial depression, hypotension, and mental disturbances (agitation, confusion, nausea, and visual loss). The clinical features are related with the severity of hyponatremia. This is a rare complication but a serious one [8]. The prevention hallmarks are carefully surgical practice and minimizing the resection time. The treatment consists of furosemide administration and hypertonic sodium solution administration. Due to our relatively small number of patients, we encountered no laboratory confirmed TURP syndrome.

Another problem is post-TURP infections. Bacteremia can usually be asymptomatic and treated using antibiotics against gram negative and gram positive bacteria. The clinical scenario is determined by chills, fever, and occasionally hemodynamic and respiratory decompensation. We faced an infection incidence of $39 \%$, an epididimitis rate of $7.11 \%$. These complications rates are similar to the other studies despite of our experience and small patients' number. The post-TURP complications are in majority preoperative period related. Preoperative bladder catheterization is one of the most important contributors in postoperative infection, but several factors are already identified as the surgical time over 90 minutes and postoperative catheter drainage failure. Recently has been reported an incidence of infection approximately $21.6 \%$ as found in a prestigious multicentre study [9]. The differences between our study and the other studies regarding the infection rate can be explained by our patients' serial. Most of our patients were previously catheterized, and it is well understood a higher rate of infection. The infection rate remains one of our challenges.

Among postoperative complications, incontinence is a big issue. Postoperative incontinence can be divided in early incontinence and urge incontinence. This complication is usually prognostic benign, but presents a problem to the patient because of patient's fear and a reduced life's quality. We report an incidence of $40 \%$ of early urge incontinence and $0.35 \%$ of total incontinence. This complication is anatomic related such as postoperative healing and the instability of the detrusor muscle. Several authors described many possible causes of incontinence. These mechanisms include sphincter failure, destrusor muscle incompetence, strictures, bladder neck hyperactivity, and residual tissue [10].

Urethral strictures remain a late postoperative problem. The patients are usually presented to the urologist for meatal strictures and/or deeper strictures (bulbar position) associated with voiding difficulties. The main surgical reason is discrepancies between instruments/catheter size and urethral diameter. In our study the presence of infection and the multiplex previously urinary catheter insertions are the main mechanisms that can explain a higher stricture incidence than reported in literature. Our stricture rate was $10.2 \%$ whereas the previous study reported an incidence that varies among $2.2 \%$ and $9.8 \%[11]$.

Regarding to the perioperative mortality and morbidity, we indentified no postoperative death. The recently reported incidence is $0-0.25 \%$ in large studies [12]. Other authors reported a 30 -day mortality incidence $0.2-0.8 \%$ [13]. Several factors are identified as risk factors including age older than 80 , large prostates, and the resection time more than 90 minutes [13]. Anesthesia technique does not influence of mortality rates $[14,15]$. Our study is patients' number limited and this explains that no death is memorized despite of comparable average patients' age. High risk patients were carefully evaluated by a multidisciplinary team composed by internal physicians, anesthesiologist, and the surgeon as well. A detailed perioperative plan, a strong cooperation between the anesthesiologist and the surgeon can guarantee the success. A skillful surgeon is an important issue in an uneventful surgical procedure.

\section{Conclusions}

Despite that TURP presents the gold standard of BHP, several complications can be faced. Major complications include bleeding, TURP syndrome, infections, and incontinence. We found few discrepancies to the other studies mainly due to limited number of the patients. We think that respecting the indications of TURP, minimizing the resection time, and a strong collaboration between the team members can reduce the complications rate and improve the patients' life quality.

\section{References}

[1] Madersbacher S, Lackner J, Bro“ ssner C, Ro“ hlich M, Stancik I, Willinger M, Schatzl G. Reoperation, myocardial infarction and mortality after transurethral and open prostatectomy: a nation-wide, long-term analysis of 23,123 cases. Eur Urol 2005; 47:499-504.

[2] Westenberg A, Gilling P, Kennett K, Frampton C, Fraundorfer $\mathrm{M}$. Homium laser resection of the prostate versus transurethral resection of the prostate: results of a randomized trial with 4year minimum long-term followup. J Urol 2004; 172:616-9.

[3] Keoghane SR, Sullivan ME, Doll HA, Kourambas J, Cranston DW. Five year data from the Oxford laser prostatectomy trial. BJU Int 2000; 86:227-8.

[4] Bachmann A, Ruszat R, Wyler S, Reich O, Seifert HH, Mu“ ller A, Susler T. Photoselective vaporization of the prostate: The Basel experience after 108 procedures. Eur Urol 2005; 47:798-804.

[5] Zwergel U. Benignes Prostathyperplasie-(BPH)-Syndrom. Operative und interventionelle Therapieoptionen. Urologe A 2001; 40:319-29.

[6] Rassweiler J, Teber D, Kuntz R, Hofmann R. Complications of transurethral resection of the prostate (TURP)-Incidence, management, and prevention. European Urology 2006; 50: 969-980.

[7] Hoffmann R. Transurethrale rezection (TURP) und transurethrale incision der Prostat. In Hoffmann R, editor. Endoskopische Urologie. Heidelberg: Springer; 2005. P 50-84.

[8] Heidler H. Frequency and causes of fluid absorption: a comparison of three techniques for resection of the prostate under continuous monitoring. BJU Int 1999; 83:619-22. 
[9] Colau A, Lucet J, Rufat P, Botto H, Benoit G, Jardin A. Incidence and risk factors of bacteria after transurethral resection of the prostate. Eur Urol 2001; 39:272-6.

[10] Hubner W, Schlarp OM. Treatment of incontinence after prostatectomy using a new minimally invasive device: adjustable continence therapy. BJU Int 2005; 96:587-94.

[11] Faul P Video-TUR:raising the gold standard. Eur Urol 1993; 24:256-61.

[12] Horninger W, Unterlechner H, Strasser H, Bartsch G. Transurethral prostatectomy: mortality and morbidity. Prostate $1996 ; 28: 195-8$.
[13] Mebust WK, Holtgreive HL, Cockett AT, Peters PC. Transurethral prostatectomy-immediate and postoperative complications: A comparative study of 13 participating institutions evaluating 3885 patients. J Urol 1989; 141:243247.

[14] Cullen DJ, Apolone G, Grenfield S, et al. ASA physical status and age predict morbidity after three surgical procedures. Ann Surg 1994; 220:3.

[15] Malhotra V. Transurethral resection of the prostate. Anesthesiol Clin North Am 2000; 18: 883-897. 